\title{
Factores que determinan la participación en el tamizaje de cáncer cervical en el estado de Morelos*
}

\author{
Eduardo César Lazcano-Ponce, Dr. S.P., ${ }^{(1)}$ Sue Moss, PhD, ${ }^{(2)}$ Aurelio Cruz-Valdez, M. en C., ${ }^{(1)}$ \\ Patricia Alonso de Ruiz, M.C., ${ }^{(3)}$ Salvador Casares-Q ueralt, M.C., ${ }^{(4)}$ Carlos Javier Martínez-León, M.C., ${ }^{(4)}$ \\ Mauricio Hernández-Avila, PhD.(1)
}

\begin{abstract}
Lazcano-Ponce EC, Moss S, Cruz-Valdez A, Alonso de Ruiz P, Casares-Queralt S, Martínez-León CJ, Hernández-Avila M. Factores que determinan la participación en el tamizaje de cáncer cervical en el estado de Morelos. Salud Publica Mex 1999;41:278-285.
\end{abstract}

\section{Resumen}

Objetivo. Determinar los principales factores relacionados con la participación en un programa de detección o portuna de cáncer (DOC) en una población con elevada mortalidad por cáncer cervical (CACU). Material y métodos En el estado de Morelos se desarrolló un estudio con base poblacional que incluyó 3197 mujeres de entre 15 y 49 años de edad, seleccionadas aleatoriamente de un marco muestral de viviendas de los 33 municipios que componen dicha entidad. Resultados El conocimiento de la utilidad de la prueba de Papanicolaou (Pap) (RM 29.6, IC 95\% 23.637) y la historia previa de síntomas ginecológicos (RM 1.7; IC 95\% 1.2-2.4) predisponen a utilizar el programa de DO C. Los factores asociados a la utilización de la prueba de Pap fueron que las mujeres tuvieran antecedentes en la utilización de uno (RM 1.4; IC 95\% 1.1-1.8), dos o más métodos de planificación familiar (RM 2.1; IC 95\% 1.6-2.8). Conclusiones. En el estado de Morelos, México, el tamizaje en CACU se ofrece predominantemente en forma oportunista, por lo que el antecedente de utilización de los servicios de salud constituye el principal determinante para la utilización del programa de DOC. Estos resultados revelan la necesidad

\author{
Lazcano-Ponce EC, Moss S, Cruz-Valdez A, \\ Alonso de Ruiz P, Casares-Queralt $S$, \\ Martínez-León CJ, Hernández-Avila M. \\ Factors which determine participation \\ in an early detection program of cervical cancer \\ in the state of Morelos. \\ Salud Publica Mex 1999;41:278-285.
}

\begin{abstract}
A bstract
Objective. To determine the main factors associated to participation in an early detection program of cancer (DPC), in a population with a high cervical cancer $(C C)$ mortality rate. Material and methods $A$ population-based, crosssectional study was performed in the state of Morelos, which included 3197 women aged between 15 and 49 years, randomly selected from a household sample frame of the 33 municipalities of the state. Results. Awareness of the utility of the Papanicolau (Pap) test (O R29.6, 95\% CI 23.6$37)$ and a former history of gynecological symptoms (OR1.7, $95 \% \mathrm{CI} 1.2-2.4)$ predisposed to greater use of the DPC. Factors associated to the use of the Pap test were precedents of using one contraceptive (O R1.4, 95\% Cl 1.1-1.8) or two or more contraceptives (O R2.1,95\% CI 1.6-2.8). Conclusions. In the sate of Morelos, Mexico, screening for cervical cancer is offered opportunistically in the context of health care use. Therefore, the precedent of using health care services is the main determining factor for use of the DPC program.These results reveal the need to design alternative strategies to promote participation among women who have no access to health services, since they
\end{abstract}

* Este estudio fue financiado por el Consejo N acional de Ciencia y Tecnología de México a través del proyecto N 0. 4939-M.A simismo, este trabajo se realizó gracias a una beca de la Unión Internacional Contra el Cáncer (International C ancer Technology Transfer Fellowship IC RETT N 0.587, 1997).

(1) Centro de Investigación en Salud Poblacional, Instituto N acional de Salud Pública, México.

(2) Cancer Screening Evaluation Unit of Institute of the Cancer Research, Reino Unido.

(3) Unidad de Patología, Universidad N acional Autónoma de México. Hospital General de la Ciudad de México.

(4) Secretaría de Salud del Estado de Morelos, México.

Fecha de recibido: 10 de julio de 1998 - Fecha de aprobado: 13 de mayo de 1999

Solicitud de sobretiros: Dr. Eduardo César Lazcano-Ponce. Centro de Investigación en Salud Poblacional. Instituto N acional de Salud Pública. Av. Universidad 655, colonia Santa María A huacatitlán, 62508 Cuernavaca, Morelos, México.

Correo electrónico: elazcano@ insp3.insp.mx 
de establecer estrategias alternativas para integrar a las mujeres que no tienen acceso a dichos servicios de atención médica, ya que constituyen el grupo de más alto riesgo para contraer CACU.

Palabras clave: neoplasmas del cuello uterino; detección o portuna de cáncer; México constitute the population group at highest risk of developing CC.

Key words: cervix neoplasms; early cancer detection; Mexico
E 1 cáncer cervical (CACU) es el tipo más común de neoplasias entre las mujeres de Latinoamérica y el Caribe. De hecho, las tasas de incidencia más altas de CACU en el mundo corresponden a los países de esa región; en la mayoría, las tasas de mortalidad por CACU han permanecido estables durante los últimos 30 años. ${ }^{1,2}$ Desde 1974 existe en México un programa nacional de detección oportuna de cáncer cervical (DOC); sin embargo, el número de muertes por CACU notificado oficialmente entre 1980 y 1997 fue cercano a 70000 , con una tasa de mortalidad de 12.5 por cada 100000 mujeres. $^{3}$ El estado de Morelos en México no es la excepción, y se estima una tasa de mortalidad por CACU de 13 por cada 100000 mujeres, que es mayor a la media nacional.

La correlación entre la disminución de la mortalidad por CACU y la puesta en marcha de programas de DOC en los países desarrollados sugiere que, en Latinoamérica, existen diversos problemas en el establecimiento de dichos programas que dan lugar a las elevadas tasas de mortalidad por ese padecimiento. En esta región han tenido éxito diversos programas poblacionales de planificación familiar y control prenatal, de manera predominante en mujeres menores de 30 años, que constituyen una alternativa indirecta para la DOC. No obstante, no ha habido la suficiente incidencia en las mujeres de mayor edad, que constituyen el grupo de más alto riesgo de contraer CACU. ${ }^{4}$

En este contexto, se han identificado varios problemas asociados con la reducida eficiencia y efectividad del programa de DOC. En México, las deficiencias se relacionan con la baja cobertura y calidad del servicio: la obtención de especímenes para realizar citología vaginal exfoliativa por técnica de Papanicolaou (Pap) es deficiente, ${ }^{5}$ los índices de diagnóstico de falsos negativos en centros de lectura de citología ginecológica son muy elevados, ${ }^{6}$ las mujeres acuden al servicio para detección tardíamente ${ }^{7}$ y existe una cobertura baja del programa de DOC en áreas rurales. ${ }^{8}$

En este artículo se identifican algunos de los factores ambientales determinantes de la participación en un programa de tamizaje poblacional para detección oportuna de CACU en un grupo de mujeres en edad reproductiva del estado de Morelos

Los resultados obtenidos permitirán llevar a cabo la comparación con estudios similares realizados en otras regiones de México, ${ }^{8}$ además de que proporcionarán información útil para la planeación de estrategias para aumentar la cobertura de mujeres con riesgo elevado de CACU.

\section{Material y métodos}

Entre 1996 y 1997 se desarrolló un estudio transversal con base en una muestra probabilística de mujeres de entre 15 y 49 años de edad, con una residencia de al menos un año en el estado de Morelos, México. Antes del estudio se estableció un marco muestral de viviendas de los 33 municipios que conforman el estado. La unidad de muestreo consistió en 3725 hogares seleccionados aleatoriamente del marco muestral previamente establecido. Para calcular el tamaño de muestra se estimó un $15 \%$ de prevalencia del antecedente de prueba de Pap anual en la población de estudio. El error de muestreo fue estimado en un 30\%, con niveles de confianza del $95 \%$. Estos hogares fueron visitados por entrevistadoras que obtuvieron un listado de las mujeres que vivían en ellos, aplicaron un cuestionario para obtener información sobre características de la vivienda (entre otros, material de construcción, disponibilidad de agua potable y drenaje), número de habitantes, género, edad y tiempo de residencia.

De cada vivienda seleccionada sólo se eligió una mujer; en los hogares donde había más de una mujer elegible, se consideró a la que figuraba como jefa del hogar, o esposa del jefe de familia para participar en el estudio. La respuesta fue positiva en $88.22 \%$ de las viviendas visitadas; en $7.5 \%$ no hubo mujeres elegibles, quedando como muestra final 3197 mujeres.

Se decidió incluir mujeres de este intervalo de edades por las siguientes razones: a) son un grupo en edad reproductiva; b) en México, los casos de CACU 
se observan después de los 20 años de edad y la frecuencia más alta de la enfermedad se presenta entre los 35 y 44 años.

Todas las mujeres entrevistadas contestaron un cuestionario con información sobre características sociodemográficas y factores de riesgo reproductivo asociados a CACU, incluyendo historia de vida sexual, antecedentes de uso de métodos anticonceptivos y razones para la utilización de la prueba de Pap.

Respecto a esto último, se incluyeron las siguientes variables: a) utilizar los servicios de salud (en particular el antecedente de uso de servicios de planificación familiar, de servicios primarios de atención médica y de control prenatal); b) acudir al programa de detección por la presencia de algún síntoma (urinario, abdominal o ginecológico), y, finalmente, c) acudir al programa de detección de forma espontánea, entre otras.

Para la exploración y el análisis de la base de datos se utilizó el paquete estadístico Stata. ${ }^{9}$ Las variables de escala continua se evaluaron en términos de su distribución original y posteriormente se categorizaron. La unidad de observación para su análisis se clasificó en mujeres con antecedente de, al menos, una prueba de Pap en su vida, y en aquellas que no tenían antecedente de tamizaje para CACU.

En la sección de antecedentes de utilización del programa de DOC, la primera pregunta fue: " $¿$ Usted sabe en qué consiste la prueba de Papanicolaou?". A las mujeres que respondieron afirmativamente se les pidió que indicaran cuál es la utilidad de esta prueba. Gracias al entrenamiento previo las entrevistadoras conocían la respuesta correcta, y después de aplicar el cuestionario explicaron a las mujeres que la prueba de Pap es para detectar oportunamente lesiones precursoras de CACU.

Se crearon dos índices: a) nivel socioeconómico y b) riesgo reproductivo; ambos fueron validados interna y externamente. En este contexto, para evaluar índices clínicos se utilizó el método clinimétrico que puede ser considerado como una medida del fenómeno clínico que identifica el estado de una entidad particular en un punto simple del tiempo. Se incluyeron las variables que reunieran dos atributos principales: evidencia básica de asociación y coherencia de componentes. Por esta razón se utilizó una prueba de consistencia interna en cada uno de los índices construidos (prueba de Wald $<0.05$ ). La validación externa del índice de riesgo reproductivo asociado a cáncer cervical se llevó a cabo con las variables referidas en un estudio de 629 casos de cáncer cervical invasor, y 1005 con- troles poblacionales, donde se observó una tendencia lineal significativa de riesgo $\left(\chi^{2} 144.2, p<0.001\right)$.

Un índice de nivel socioeconómico se construyó de manera similar a la que describieron Bronfman y colaboradores ${ }^{10}$ a partir de la información de seis variables socioeconómicas. En primer lugar, se construyó un índice de nivel de hacinamiento a partir de las variables "número de personas que viven en la vivienda" y "número de cuartos en la vivienda sin contar con baño y cocina". Este índice, junto con las variables "material del piso de la vivienda", "disponsibilidad de agua potable" y "forma de eliminación de excretas", da lugar al índice de condiciones de la vivienda. Finalmente, el índice de nivel socioeconómico se construye mediante el índice de condiciones de la vivienda y la escolaridad del jefe de familia. Tal cual lo informan los autores, el índice es ordinal y tricotómico, y es útil para poblaciones que son homogéneas, como es la población de estudio.

Para evaluar la asociación entre el riesgo de CACU y el uso de la prueba de Pap se construyó un índice de variables reproductivas con aquellas que se han asociado significativamente a CACU en estudios realizados previamente en México. ${ }^{11}$ Estas variables fueron: número de partos vaginales (de $0 \mathrm{a} \leq 10$ ); edad de inicio de la vida sexual (de $\leq 15 \mathrm{a} \geq 24)$; número de parejas sexuales (de 1 a $\leq 10$ ) y edad (en categorías de cinco años, $\leq 25,26$ a 30, 31 a 35,36 a $40, y \geq 41$ ). El índice se validó interna y externamente. ${ }^{12}$

Para evaluar la asociación entre la utilización de la prueba de Pap y las variables sociodemográficas, el nivel socioeconómico y las variables reproductivas, se obtuvieron las razones de momios (RM) y los intervalos de confianza al $95 \%$ (IC95\%) por medio de modelos de regresión logística no condicional, y se utilizaron modelos bivariados y multivariados. El modelo final quedó integrado con las variables que tuvieron una asociación significativa, incluyendo el índice de riesgo reproductivo de CACU ya que, de acuerdo con estudios previos, éste incluye los principales factores de riesgo de este padecimiento en mujeres mexicanas. Para controlar las posibles variables de confusión se utilizó la regresión logística múltiple.

\section{Resultados}

En el cuadro I se presenta la información básica sobre las características sociodemográficas, socioeconómicas y reproductivas de las usuarias y las no usuarias de los servicios del programa de DOC. La media de edad es mayor en usuarias (media $=35.6, \mathrm{DE}=7.7$ ), en com- 
Cuadro I

Características sociodemográficas y factores

DE RIESGO DE CÁNCER CERVICAL EN MUJERES Del estado de Morelos. México, 1996-1997

\begin{tabular}{|c|c|c|c|c|c|}
\hline \multirow[b]{2}{*}{ Variables } & \multicolumn{2}{|c|}{$\begin{array}{l}\text { Usuarias } \\
\text { de DOC }\end{array}$} & \multicolumn{2}{|c|}{$\begin{array}{l}\text { No usuarias } \\
\text { de DOC }\end{array}$} & \multirow[b]{2}{*}{ t-test } \\
\hline & Media & $\mathrm{DE}$ & Media & $\mathrm{DE}$ & \\
\hline Edad & 35.6 & 7.74 & 28.2 & 8.18 & $<0.05^{*}$ \\
\hline Años de escolaridad (mujeres) & 6.7 & 1.1 & 6.8 & 0.9 & $>0.05$ \\
\hline \multicolumn{6}{|l|}{ Años de escolaridad (parejas } \\
\hline masculinas) & 6.2 & 1.2 & 5.3 & 0.7 & $<0.05^{*}$ \\
\hline Menarca & 13.1 & 1.3 & 13.1 & 1.4 & $>0.05$ \\
\hline Embarazos & 4.0 & 2.3 & 3 & 2.3 & $<0.05^{*}$ \\
\hline Edad del ler. parto & 19.4 & 3.8 & 19.5 & 3.9 & $>0.05$ \\
\hline Hijos vivos & 3.2 & 2 & 2.2 & 2 & $<0.05^{*}$ \\
\hline Cesáreas & 0.3 & 0.1 & 0.3 & 0.1 & $>0.05$ \\
\hline Abortos & 0.7 & 0.2 & 0.3 & 0.1 & $<0.05^{*}$ \\
\hline Edad de inicio de vida sexual & 18.4 & 3.5 & 18.4 & 3.4 & $>0.05$ \\
\hline Número de parejas sexuales & 1.4 & 0.9 & 1.3 & 0.8 & $>0.05$ \\
\hline Indice de riesgo reproductivo & 15.9 & 4.8 & 13.4 & 4.8 & $<0.05$ \\
\hline Historia de síntomas generales & 4.2 & 2.7 & 3.6 & 2.7 & $<0.05$ \\
\hline Historia de síntomas ginecológicos & 1.6 & 1.3 & 1.5 & 1.3 & $<0.05$ \\
\hline $\begin{array}{l}\text { Historia de uso de } \\
\text { anticonceptivos }\end{array}$ & 1.6 & 1.1 & 0.9 & 0.5 & 0.05 \\
\hline
\end{tabular}

* Significancia estadística $p<0.05$

paración con la de las no usuarias (media $=28.2, \mathrm{DE}=$ 8.1). Asimismo, la educación del jefe de familia, el número de embarazos, de partos vaginales y de abortos, es significativamente más alto en las usuarias de la prueba de Pap.

Como se observa en la figura 1, 63.3\% de las mujeres entrevistadas tenían el antecedente de haberse sometido al menos a una prueba de Pap en su vida. A este respecto, $21.6 \%$ tenían una sola prueba de tamizaje realizada; $10 \%$ de la población tenía dos pruebas de tamizaje previas. Finalmente, $32 \%$ de las mujeres de entre 15 y 49 años del estado de Morelos tuvieron el antecedente de haberse sometido a tres o más pruebas de Pap.

La prevalencia del antecedente de Pap en mujeres entre 45 y 49 años de edad es 4.54 veces mayor (IC95\% 3.3-6.2), en comparación con las menores de 20 años (cuadro II).

El conocimiento de la utilidad de la prueba de Pap se asocia estrechamente con la utilización del programa de DOC en el estado de Morelos (RM 29.6, IC95\% 23.6-37) y el antecedente de haber presentado síntomas ginecológicos incrementa la prevalencia de utilización del tamizaje (RM 1.7, IC95\% 1.2-2.4). Las mujeres con historia de utilización de uno (RM 1.4,

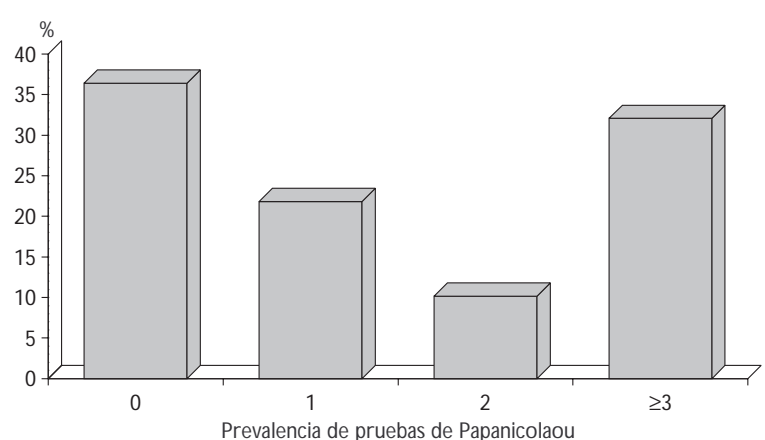

Figura 1. Prevalencia de tamizaje para cáncer Cervical en el estado de Morelos. México, 1996-1997

\section{Cuadro II \\ Prevalencia de uso de la prueba de Pap por GRUPOS DE EDAD EN EL ESTADO DE MoRelos. MÉXICO, 1996-1997}

Grupos de edad Usuarias No usuarias RM* IC95\%

\begin{tabular}{lrrrr} 
15-19 años & 23 & 132 & 0.16 & $0.09-0.25$ \\
\hline $20-24$ años & 155 & 334 & 0.41 & $0.32-0.53$ \\
\hline $25-29$ años & 303 & 270 & 1.00 & \\
\hline $30-34$ años & 444 & 161 & 2.46 & $1.93-3.13$ \\
\hline $35-39$ años & 413 & 130 & 2.83 & $2.20-3.70$ \\
\hline $40-44$ años & 375 & 73 & 4.60 & $3.40-6.20$ \\
\hline $45-49$ años & 321 & 63 & 4.54 & $3.31-6.23$ \\
\hline Prueba de tendencia & & & & $p<0.05$
\end{tabular}

* Razón de momios ajustada por edad, conocimiento de la utilidad de la prueba de Pap, historia de síntomas durante los seis meses previos, historia de uso de métodos de planificación familiar, índice de riesgo reproductivo y derecho a seguridad social

IC 95\% 1.1-1.8), dos o más métodos de planificación familiar (RM 2.1, IC95\% 1.6-2.8) están relacionadas más estrechamente con el programa de DOC. Asimismo, a las mujeres con el antecedente de haberse sometido al menos a una prueba de Pap, corresponde una mayor prevalencia de utilización del tamizaje en la medida en que se incrementa el índice de riesgo reproductivo para CACU (en el quintil cinco del índice de riesgo RM 6.6, IC95\% 4.6-9.5) (cuadro III).

Existe una relación de tendencia significativa entre la historia de los síntomas abdominales y ginecológicos y la utilización de la prueba de Pap $(p<0.05)$ : en las mujeres con una historia de cuatro síntomas la prevalencia de utilización del tamizaje es mayor (RM 2.06, IC95\% 1.6-2.6). Así, el antecedente de 13 o más años de 
Cuadro III

Determinantes de participación en el tamizaje De CÁnCer Cervical en el estado de Morelos. MÉXıCO, 1996-1997

Usuarias No usuarias $\quad$ RM* IC95\%

Conocimiento de la utilidad de la prueba de Papanicolaou

\begin{tabular}{lrrrr} 
No & 193 & 869 & 1.00 & \\
\hline Sí & 1832 & 279 & 29.6 & $23.6-37.0$
\end{tabular}

Historia de síntomas ginecológicos seis meses previos a la entrevista

\begin{tabular}{rrrrr} 
No & 147 & 156 & 1.00 & \\
\hline Sí & 1887 & 1007 & 1.7 & $1.2-2.4$
\end{tabular}

Historia de utilización de métodos de planificación familiar Métodos

\begin{tabular}{|c|c|c|c|c|}
\hline No & 409 & 449 & 1.00 & \\
\hline 1 & 746 & 451 & 1.4 & $1.1-1.8$ \\
\hline$>=2$ & 975 & 288 & 2.1 & $1.6-2.8$ \\
\hline
\end{tabular}

Indice de riesgo reproductivo

\begin{tabular}{lllll}
1 quintil & 380 & 441 & 1.00 & \\
\hline 2 & 288 & 195 & 1.6 & $1.2-2.2$ \\
\hline 3 & 438 & 204 & 2.3 & $1.7-3.2$ \\
\hline 4 & 534 & 167 & 4.0 & $2.9-5.5$ \\
\hline 5 & 357 & 110 & 6.6 & $4.6-9.5$ \\
\hline \multicolumn{4}{l}{ Prueba de tendencia }
\end{tabular}

Seguridad social

\begin{tabular}{lrrll} 
No & 1529 & 978 & 1.00 & \\
\hline Sí & 553 & 192 & 1.5 & $1.1-2.0$
\end{tabular}

* Razón de momios ajustada por todas las variables incluidas

escolaridad del jefe de familia incrementa la prevalencia de utilización de la prueba de Pap (RM 2.5, IC95\% 1.7-3.6) (cuadro IV).

En resumen, los principales factores que determinan la utilización de la prueba de Pap en el estado de Morelos son: el conocimiento de su utilidad; la historia de síntomas ginecológicos, abdominales y urinarios, y el antecedente de la utilización de métodos de planificación familiar.

\section{Discusión}

En este estudio se observa una relación directamente proporcional entre la prevalencia de utilización de la prueba de Pap y el nivel de escolaridad de las mujeres y sus parejas sexuales. Por esa razón, el factor prin-

\section{Cuadro IV \\ EsCOLARIDAD Y UTILIZACIÓN DEL PROGRAMA DE DETECCIÓN OPORTUNA DE CÁNCER EN EL ESTADO De Morelos. México, 1996-1997}

Escolaridad (años) Usuarias No usuarias RM* IC95\%

Escolaridad en mujeres que utilizan tamizaje para cáncer cervical

\begin{tabular}{|c|c|c|c|c|}
\hline Cero & 163 & 101 & 1.00 & \\
\hline $1-6$ & 997 & 523 & 1.2 & $0.9-1.5$ \\
\hline $7-9$ & 494 & 359 & 0.8 & $0.6-1.1$ \\
\hline $10-12$ & 238 & 125 & 1.1 & $0.8-1.6$ \\
\hline$>12$ & 141 & 54 & 1.6 & $1.1-2.4$ \\
\hline
\end{tabular}

Escolaridad de la pareja sexual masculina

\begin{tabular}{|c|c|c|c|c|}
\hline Cero & 222 & 162 & 1.00 & \\
\hline $1-6$ & 910 & 506 & 1.3 & $1.0-1.6$ \\
\hline $7-9$ & 477 & 303 & 1.5 & $0.9-1.5$ \\
\hline $10-12$ & 212 & 121 & 1.3 & $0.9-1.7$ \\
\hline$>12$ & 180 & 52 & 2.5 & $1.7-3.6$ \\
\hline
\end{tabular}

* Razón de momios ajustada por edad, conocimiento de la utilidad de la prueba de Pap, historia de síntomas durante los seis meses previos, historia de uso de métodos de planificación familiar, índice de riesgo reproductivo y derecho a seguridad social

cipal para utilizar el programa de detección de CACU es el conocimiento de la utilidad de la prueba de Pap. Este hallazgo es consistente y similar con los que se han encontrado en estudios previos de base poblacional en un área predominantemente urbana (Ciudad de México), y en una área rural del estado de Oaxaca, utilizando el mismo diseño de estudio y los mismos instrumentos para recolectar la información. La principal diferencia con lo que se ha informado previamente en México reside en que, en el estado de Morelos, el tamizaje ocurre en forma oportunista y no espontánea, es decir que la mayor prevalencia de utilización de la prueba de Pap se observa en las usuarias de los servicios de salud, predominantemente en aquellas que acuden a los módulos de planificación familiar y de control prenatal y en las derechohabientes del sistema de seguridad social. En ese contexto cabe destacar que uno de los principales factores para utilizar el Pap es la presencia o antecedente de un síntoma ginecológico, urinario o abdominal, aunque la premisa básica del estudio es que se lleve a cabo periódicamente en mujeres asintomáticas. En consideración a lo anterior, la educación comunitaria para fomentar el uso de la prueba de Pap es una de las principales acciones que deben llevarse a cabo con las mujeres que corren un gran riesgo de padecer neoplasia cervical. 
Las decisiones que toma la población respecto a su salud -como la búsqueda de atención por enfermedad- se basan en sus creencias y actitudes personales, mismas que se explican a través de numerosos factores tales como experiencias previas de enfermedad, tanto personales como familiares; percepción de la asistencia profesional; otros contactos con profesionales de la salud; información recibida, y consejos de familiares, amigos y del propio médico. ${ }^{13}$ Algunas veces, las creencias personales acerca de las enfermedades pueden ser muy idiosincráticas. ${ }^{14}$ En ese contexto, se han identificado los principales factores que predicen la utilización del programa de DOC en México ${ }^{8}$ y en otros países. Estos factores incluyen las actitudes y creencias acerca del Pap, ${ }^{15}$ los beneficios percibidos sobre la efectividad del programa de DOC y el apoyo significativo de la familia, las amistades, la comunidad y los servicios de salud.

En México y entre las mujeres étnicamente marginadas de los países desarrollados, la solución del problema implica mantener elevados los niveles de cobertura en la población blanco, sin desperdiciar recursos en pruebas frecuentes de Pap en mujeres con bajo riesgo. Los hallazgos de este estudio, realizado en áreas suburbanas del estado de Morelos, sugieren que la probabilidad para que las mujeres de entre 15 y 49 años de edad participen en un programa así está determinada por la frecuencia con la que se utilizan los servicios de salud, particularmente los de planificación familiar, y por la presencia de algún síntoma abdominal o ginecológico más que por su riesgo de sufrir la enfermedad o por incluirse espontáneamente en el programa.

Como se ha notificado previamente en México, ${ }^{7}$ la utilización de la prueba de Pap brinda un efecto protector cuando las mujeres acuden a realizársela en forma espontánea, sin la presencia de síntomas. Los factores que influyen en la correcta aplicación del programa de DOC por parte de los profesionales de la salud incluyen la habilidad para aconsejar acerca de la importancia del tamizaje y la promoción del estudio en mujeres asintomáticas. ${ }^{16} \mathrm{~A}$ diferencia del hallazgo en Morelos, en una evaluación del programa de DOC en Australia, la prevalencia de síntomas en las usuarias fue muy baja: $6 \%$ de las mujeres presentaron leucorrea; 5\%, sangrado anormal, y 10\% acudieron motivadas por un Pap anormal previo. Asimismo, durante un periodo de 10 años, del total de estudios de Pap realizados en mujeres australianas, sólo $20.9 \%$ correspondieron a tamizaje oportunista relacionado con usuarias de anticonceptivos hormonales, y $8 \%$ a mujeres que acudieron a control prenatal.

Cabe subrayar que la conducta de prevención secundaria de la salud se define como las acciones diri- gidas hacia la detección de la enfermedad en un estadio asintomático. Cuando se detecta y se trata una neoplasia cervical en el estadio preinvasivo, la cura es de 95\% o más. ${ }^{17}$ En comparación con el estado de Morelos, México, en Islandia, donde la incidencia y el porcentaje de mortalidad por CACU se encuentran entre los más bajos del mundo, la participación de mujeres asintomáticas en los programas de DOC es alta. En un estudio llevado a cabo en la ciudad de Hafnarfjordur, Islandia, $89.3 \%$ de las mujeres habían utilizado el programa de DOC durante los cinco años previos y sólo $4.1 \%$ nunca se habían realizado una prueba de Pap. En esta población, la histerectomía fue la razón más común para no participar. ${ }^{18}$

A este respecto, en Suecia, donde se promueve la educación para la salud entre la población, existe otra concepción de los servicios preventivos. Así, con una elevada cobertura de tamizaje para $\mathrm{CACU}$, por medio de folletos y artículos se enfatiza que la mujer que presente un sangrado anormal o la presencia de algún tipo de fluido, también anormal, particularmente después de haber tenido relaciones sexuales, debe buscar atención médica; de esa manera, se incide predominantemente en mujeres de alto riesgo de enfermedad. Asimismo, se considera que la sensibilización acerca del cáncer ginecológico y sus síntomas en el ambiente médico es un factor determinante para la participación en los programas de DOC. ${ }^{19}$

A pesar de que se ha notificado una prevalencia del uso del Pap alta entre las mujeres jóvenes en los países desarrollados y baja en los no industrializados, en el estado de Morelos, en México, el antecedente de Pap en mujeres en edad reproductiva es alta, pero no incide en mujeres de alto riesgo. A este respecto, en un estudio con mujeres en edad reproductiva en Estados Unidos de América (EUA), la tasa anual de Pap para el grupo de edad de entre 15 y 44 años fue de $67 \%{ }^{20}$ Asimismo, más de $90 \%$ de las mujeres que tuvieron una visita médica en un periodo de 12 meses se realizaron el Pap. ${ }^{20}$ Estos hallazgos son similares al 97\% de mujeres de 30-49 años que informaron haberse sometido a una prueba de Pap en Inglaterra y Gales. ${ }^{15}$

En varios estudios con mujeres en edad reproductiva, ${ }^{21}$ el nivel socioeconómico bajo se asoció con el uso poco frecuente de los servicios médicos, especialmente en las áreas rurales -como se ha informado previamente en México- ${ }^{8}$ donde la prevalencia del uso de Pap es, en general, baja. Por ejemplo, en dos años la tasa de prevalencia de Pap para mujeres mayores de 44 años nativas de Alaska, fue de $64 \% .{ }^{22}$ Un estudio poblacional en Taiwan, donde los servicios de salud son subutilizados, mostró que $40 \%$ de las mujeres muestreadas nunca se habían realizado un Pap y $86 \%$ 
no notificó ni uno solo el año anterior. Además, las mujeres de nivel escolar bajo, las no trabajadoras, las casadas y las que vivían fuera de la ciudad, recurrieron muy poco al programa de DOC. ${ }^{23}$ En el estudio de Morelos, la muestra fue homogénea en cuanto al nivel socioeconómico, y la mayor prevalencia de utilización de Pap se observó en mujeres que cuentan con adscripción a los servicios de seguridad social.

Por otra parte, una investigación realizada en las ciudades de Génova y Roma, en Italia, ${ }^{24}$ mostró que en los países desarrollados el incremento en la edad es la barrera más importante para participar en los programas de tamizaje, de tal manera que deben realizarse grandes esfuerzos para involucrar a las mujeres mayores, especialmente por su elevado riesgo de adquirir el CACU; ello contrasta con el estudio realizado en Morelos, donde la prevalencia de uso de Pap se incrementó linealmente con la edad.

En las mujeres del medio rural en el este del estado de Washington, EUA, los factores determinantes del uso del programa de DOC fueron un nivel socioeconómico y una escolaridad elevados. ${ }^{25}$ En Morelos, la mayor prevalencia de utilización se observa no sólo con el incremento de la escolaridad de las mujeres estudiadas, sino también en forma significativa con el nivel de escolaridad de la pareja sexual masculina. Otro estudio, con mujeres latinas y anglosajonas en la comunidad de Orange en California, EUA, mostró que las primeras utilizan el programa de DOC con menos frecuencia que las anglosajonas debido a que las creencias y la interpretación de las enfermedades por parte de las latinas pueden influir en el uso del Pap. También se observó que estas creencias prevalecen más entre inmigrantes; la preocupación de que el CACU se transmite sexualmente puede desalentar a las inmigrantes latinas en la correcta utilización de un programa largo de DOC. ${ }^{26}$ Asimismo, un estudio acerca de las percepciones sobre el CACU y las prácticas respecto al Pap, en relación con la orientación sexual de las mujeres, mostró que la principal barrera para las usuarias no regulares del programa de DOC fue el carecer de seguro médico; ${ }^{27}$ este hecho se corroboró en el estado de Morelos, en México, donde la derechohabiencia incrementó cerca de dos veces la prevalencia de utilización de Pap.

Los resultados del estudio en Morelos indican la necesidad de llevar a cabo intervenciones educativas para incrementar la cobertura en mujeres de alto riesgo. A este respecto, varios autores ${ }^{28}$ han estudiado de manera extensa las campañas educativas para promover la participación en los programas de DOC. Dichas campañas de comunicación pública se diseñan para incrementar el número de mujeres que se hacen exámenes cervicales pero no han podido reclutar con éxito suficiente número entre los grupos étnicos marginados. En una campaña en la que se utilizó la técnica de grupos focales para aumentar el conocimiento, las actitudes positivas y los cambios de conducta de mujeres afroamericanas, se encontró que la influencia personal de los líderes de opinión, combinada con contactos interpersonales para animar al cuidado preventivo, ayudan a que las mujeres participen en los programas de DOC. ${ }^{29}$

Otros autores, a través de métodos de análisis cualitativo, también han estudiado de manera amplia las razones por las cuales las mujeres de grupos étnicos marginados no participan en los programas de DOC. Los resultados de un estudio con base en la comunidad metropolitana de Phoenix, EUA, indican que las mujeres hispanas saben menos acerca de la utilidad del Pap, y la tasa de utilización del programa de DOC es también menor, por lo que aumenta el riesgo de que se realicen el estudio en estadios avanzados de cáncer. ${ }^{30}$ En las comunidades de los estados del centro de EUA las tasas de práctica de Pap de las mujeres camboyanas son significativamente más bajas que las de las no camboyanas. Las barreras incluyen la falta de conocimientos acerca del cáncer, timidez durante el examen físico, falta de transporte, miedo ante una institución médica grande y especializada y la falta de seguimiento individual. ${ }^{31}$

Los obstáculos para avanzar en los programas de DOC incluyen la falta de prioridad que se da a las cuestiones de la salud de la mujer, el insuficiente conocimiento público sobre el CACU y un escaso entendimiento acerca de las limitaciones de la prevención secundaria. ${ }^{32}$ En las comunidades nativas se ha identificado a las mujeres mayores y a las que son jefas de familia como la población prioritaria por sus actitudes negativas, derivadas de experiencias previas, así como por la gran influencia que tienen sobre las mujeres más jóvenes. ${ }^{33}$ Entre las mujeres hispanas del estado de Washington, 78\% nunca se han realizado un Pap. En este caso, el costo se notificó como el obstáculo principal para el tamizaje. Adicionalmente, una proporción sustancial de las mujeres estudiadas percibió al Pap como innecesario o como un procedimiento de diagnóstico, más que como una medida de prevención. ${ }^{34}$

En este contexto, se deben desarrollar algunas estrategias para estimular el proceso de cambio de conducta y combinarlas con una estrategia integral para los cuidados de la salud de las mujeres, en dos contextos principales, los servicios de salud y la comunidad. En los servicios de salud, en un mismo programa se pueden incluir los servicios prenatales y los ginecológicos (la planificación familiar, las pruebas de Pap 
y la prevención de las ETS, entre otras actividades). ${ }^{35}$ En el ámbito comunitario, las actividades que han ocasionado un incremento significativo del tamizaje para CACU han sido la promoción de la salud por brigadas comunitarias de servicios alternativos, incluyendo tamizaje para enfermedades crónicas, así como la promoción de la salud materno-infantil. Por estas razones, en los países en desarrollo los programas de salud deberán ser promovidos en forma integral.

Finalmente, en las naciones que tienen bajas coberturas de tamizaje para CACU es necesario llevar a cabo mayor investigación acerca de los conocimientos, actitudes, creencias y otras barreras de utilización, que permitan postular intervenciones acordes a las necesidades de la población en riesgo.

\section{Referencias}

1. Robles $S$. Introduction to the special issue:T imely detection of cervical cancer. Bull Pan Am Health O rgan 1996;30(4):285-289.

2. Beral V, Hermon C, Muñoz N, D evesa S. Cervical cancer. Cancer Surv 1994;19/20:265-285.

3. Lazcano-Ponce E, Rascon R, Lozano R,Velasco E. Mortality from carcinoma of the uterine cervix in Mexico.Acta Cytol 1996;40:506-512.

4. Robles SC,W hite F, Peruga A. Trends in cervical cancer mortality in the Americas. Bull Pan Am Health O rgan 1996;30(4):290-301.

5. Lazcano-Ponce E, Alonso P, López L, Hernández M. Q uality control study on negative gynecological cytology in Mexico. Diagn Cytopathol1994;10: 10-14.

6. Lazcano-Ponce E, Alonso P, López L, N ájera P, Avila R, Escandón C et al.Validity and reproducibility of cytology diagnosis in a sample cervical cancer screening centers in Mexico.Acta Cytol 1997;41:227-284.

7. Hernández M, Lazcano E, López L, Alonso P, Rojas R. Evaluación del programa de detección oportuna de cáncer cervical en México.Un estudio de casos y controles con base poblacional. Gac Med Mex 1994;130(4): 201-209.

8. Lazcano-Ponce E, N ájera P, Buiatti E, Alonso P, Kuri P, Cantoral L et al. The cervical cancer screening program in Mexico. Problems with access and coverage. Cancer Causes \& Control 1997;8:698-704.

9. Stata Corporation. 1993 Stata reference manual. Release 3.1.6 a edición. College Station, TX: Stata Corporation,1993.

10. Guiscafré $H$, Bronfman M, Padilla G, Reyes CM, C astro R, Muñoz 0 et al. Estrategias para mejorar patrones terapéuticos utilizados en diarrea aguda en unidades de atención médica primaria. II. Medición de la desigualdad: una propuesta metodológica, análisis de las características socioeconómicas de la muestra. Arch Invest Med 1988;19(4): 419-425.

11. Lazcano-Ponce E, Hernández M, López L,Alonso P, G onzález G, Torres $A$ et al. Factores de riesgo reproductivo e historia de vida sexual asociados a cáncer cervical en una muestra de mujeres de la Ciudad de México. Rev Invest Clin 1995;47:377-385.

12. Kirshner B,G uyatt G.A methodological framework for assessing health indices. J Chronic D is 1985;38(11):27-36.

13. SenskyT. Eliciting lay beliefs across cultures:Principles and methodology. $\mathrm{Br}$ J Cancer 1996;74 suppl XXIX:S63-S65.
14. Sensky T. Patient's reactions to illness: Congnitive factors determine responses and are amenable to treatment. Br Med J 1990;300:622-623.

15. Luke K. Cervical cancer screening: Meeting the needs of minority ethnic women. Br J Cancer 1996;74 suppl XXIX:S47-S50.

16. Hunt SM, Macleod M. Health and behavioural change: Some lay perspectives. Community Med 1987;9:68-76.

17. Straton J, Holman C, Edwards B. Cervical cancer screening in W estern Australia in 1992:Progress since 1983. Med J Aust 1993;159:657-661.

18. Conway-K .Attitudes to Papanicolaou smears. J Psychosom 0 bstetGynecol1996;17(4):189-194.

19. Bergmann JB, Sigurdsson JA; Sigurdsson K. W hat attendance rate can be achieved for Pap smear screening? A case-control study of the characteristics of non-attenders and results of reminder efforts. Scand J Prim Health Care 1996;14(3):152-158.

20. Ponten J,A dami H, Bergstrom R, Dillner J, Friberg L, Gustafson $L$ et al. Strategies for global control of cervical cancer. Int J Cancer 1995;60:1-26. 21. W ilcox LS; Mosher W D. Factors associated with obtaining health screening among women of reproductive age. Public Health Rep 1993; 108(1):76-86.

22. Provost EM.Cervical cancer screening on theYukon-Kuskokwim D elta, SouthwestA laska:A population-based study.C ancer 1996;78(7):1598-1602. 23.W ang PD, Lin RS. Sociodemographic factors of Pap smear screening in Taiwan. Public Health 1996;110(2):123-127.

24. Bonelli L, Branca M, Ferreri M, Barizzone D, Rossi E, Cedri-S et al. Attitude of women towards early cancer detection and estimation of the compliance to a screening program for cervix and breast cancer. C ancer Detect Prev 1996;20(4):342-352.

25. Skaer TL, Robison LM, Sclar DA, Harding-GH. Cancer-screening determinants among $\mathrm{H}$ ispanic wo men using migrant health clinics.J Health Care Poor Underserved 1996;7(4):338-354.

26. Hubbell FA, Chávez LR, Mishra SI,Valdez RB. Beliefs about sexual behavior and other predictors of Papanicolaou smear screening among Latinas and Anglo women. Arch Intern Med 1996;156(20):2353-2358.

27. Price JH, Easton AN ,Telljohann SK,W allace PB. Perceptions of cervical cancer and Pap smear screening behavior by women's sexual orientation. J Community Health 1996;21(2):89-105.

28. Dignan M, Michielutte R, Blinson K, W ells HB, Case LD, Sharp P et al. Effectiveness of health education to increase screening for cervical cancer among Eastern-band Cherokee Indian women in N orth Carolina.J N atl Cancer Inst 1996;88(22):1670-1676.

29. W illiams GA, Abbott RR, Taylor DK. Using focus group methodology to develop breast cancer screening programs that recruit African American women.J Community Health 1997;22(1):45-56.

30. Harmon MP, Castro FG, Coe K. Acculturation and cervical cancer: Knowledge, beliefs and behaviors of $\mathrm{H}$ ispanic women.W omen $\mathrm{H}$ ealth 1996; 24(3):37-57.

31. Kelly AW, D el-Mar-Fores-C hacori M,W ollan PC, Trapp MA,W eaver AL, Barrier PA et al. A program to increase breast and cervical cancer screening for Cambodian women. Mayo Clin Proc 1996;71(5):437-444.

32. Cohen MM. W hy is there no progress against cervical cancer? $C$ an Med Assoc J 1996;154(12):1867-1869.

33. Strickland CJ, Chrisman NJ,Yallup M, Powell K, Squeoch MD.W alking the journey of womanhood:Yakama Indian women and Papanicolaou (Pap) test screening. Public Health N urs 1996;13(2):141-150.

34. Skaer TL, Robison LM, Sclar DA, Harding GH. Knowledge, attitudes, and patterns of cancer screening: $A$ self-report among foreign born $\mathrm{H}$ ispanic women utilizing rural migrant health clinics. J Rural Health 1996:12(3):169-177.

35. Barbosa RM, D 0-Lago TG, Kalckman S, Villela W V. Sexuality and reproductive health care in São Paulo, Brazil. Health Care Women Int 1996;17(5):413-421. 